\title{
Review Article \\ Proton-Based Stereotactic Ablative Radiotherapy in Early-Stage Non-Small-Cell Lung Cancer
}

\author{
Jonathan D. Grant and Joe Y. Chang \\ Department of Radiation Oncology, The University of Texas M.D. Anderson Cancer Center, Houston, TX 77030, USA \\ Correspondence should be addressed to Joe Y. Chang; jychang@mdanderson.org
}

Received 4 April 2014; Revised 30 May 2014; Accepted 21 June 2014; Published 17 July 2014

Academic Editor: Yusuke Demizu

Copyright ( 2014 J. D. Grant and J. Y. Chang. This is an open access article distributed under the Creative Commons Attribution License, which permits unrestricted use, distribution, and reproduction in any medium, provided the original work is properly cited.

\begin{abstract}
Stereotactic ablative radiotherapy (SABR), a recent implementation in the practice of radiation oncology, has been shown to confer high rates of local control in the treatment of early stage non-small-cell lung cancer (NSCLC). This technique, which involves limited invasive procedures and reduced treatment intervals, offers definitive treatment for patients unable or unwilling to undergo an operation. The use of protons in SABR delivery confers the added physical advantage of normal tissue sparing due to the absence of collateral radiation dose delivered to regions distal to the target. This may translate into clinical benefit and a decreased risk of clinical toxicity in patients with nearby critical structures or limited pulmonary reserve. In this review, we present the rationale for proton-based SABR, principles relating to the delivery and planning of this modality, and a summary of published clinical studies.
\end{abstract}

\section{Introduction}

Non-small-cell lung cancer (NSCLC) in its early stage can be treated definitively with favorable outcomes. The standard for curative therapy has historically been surgery for those medically able to undergo a lobectomy. However, given the frequent coincident morbidities such as elderly age, cardiac disease, and poor pulmonary function influenced by tobacco use, a significant proportion of patients are not operative candidates. Developing technologies have introduced lessinvasive methods of addressing early-stage NSCLC with curative intent, including advances in external beam radiotherapy.

\section{Stereotactic Ablative Radiotherapy}

Conventionally fractionated radiotherapy, applied for over a century, delivers a protracted dose over multiple daily treatments commonly given over 5-7 weeks. This fractionated approach allows nearby normal tissues, which receive collateral amounts of radiation, to undergo cellular and DNA damage repair, thus minimizing damage to surrounding critical structures. Unfortunately, however, conventional fractionation for lung cancers has been shown to offer limited local control [1]. As improving technology in radiation planning, delivery, and motion management has evolved, stereotactic ablative radiotherapy (SABR) has emerged as an effective treatment for early-stage NSCLC. SABR is defined as a "high dose of radiation to an extracranial target in the body using either a single dose or a small number of fractions" [2]. SABR relies on precise localization of the tumor and careful consideration of nearby critical structures to avoid high doses in the sensitive region.

Applied to peripherally located early stage tumors $<5 \mathrm{~cm}$ in size, SABR has been shown to produce a local control rate of $>90 \%$, with a low incidence of acute and long-term side effects [3]. The prescription dose strength of various radiotherapy fractionation schedules are often compared through 2-Gray biologically dose equivalent (BED) calculations. Studies have indicated that a BED of $>100$ Gray equivalents $(\mathrm{GyE})$ is correlated with improved local control and survival $[4,5]$.

SABR was initially developed using photon-based radiotherapy, consisting of high-energy X-rays. Advantages of photon SABR include widespread availability, mature clinical experience, and good outcomes in appropriately selected patients. The application of protons in delivering SABR to early-stage NSCLC has emerged as a tool which may be able to reduce the risk of toxicity in patients with complicated 
presentations [6]. The advantage of proton therapy hinges on its ability to minimize dose to normal tissues distal to the tumor. Thus, potential clinical benefit may be offered to patients with limited pulmonary reserve, tumors in close geometric proximity to critical normal structures, or in patients who have received prior thoracic radiation $[7,8]$. In these cases, reducing radiation damage to normal tissues is an absolute priority. The physical properties of protons, along with a discussion of technical issues related to delivery, planning considerations, and published clinical studies, will be presented herein.

\section{Physical and Biologic Properties of Proton Therapy}

Proton therapy is the most widespread application of charged particles for treatment of tumors in the body. Carbon ion therapy, used in several centers in Europe and Asia, will not be addressed in this review. Proton therapy was initially developed and clinically implemented in the mid-twentieth century, but it was limited to the treatment of ocular and intracranial tumors. These locations present minimal motion challenges and high fidelity with planning calculations. With improving technologies over the last several decades, the application of proton therapy has expanded to tumors throughout the entire body. Over 30 proton beam facilities are in operation worldwide, with many more being under construction and being expected to open over the next several years.

The defining characteristic of proton therapy is the "Bragg peak," a description of the high-energy dose deposition over a small distance at the end of the proton range (Figure 1). Proximal to this peak is a low entrance dose profile, within minimal or no radiation dose to distal tissues. The range of a proton is inversely proportional to the density of the tissue. In contrast, photon-based radiotherapy delivers its peak energy within several centimeters of tissue penetration, followed by a gradual dose decline, resulting in a penetration of $\mathrm{X}$ rays through the body with a larger area of tissue exposed to collateral radiation (Figure 1). This improved selectivity in radiation dose delivery may help reduce normal tissue toxicity, allow for higher dose delivery to tumor, and reduce the chance of secondary late malignancies.

Biologic differences also exist between protons and photons, with regard to how these different types of radiation interact with living cells. The nature of these effects and implications on tumor control and normal tissue toxicity is largely unknown at the present time and represents an area of growing research. Protons have been found to have a higher linear energy transfer (LET) than photons, which quantifies the magnitude of DNA damage from reactive ions. This LET effect also increases near the distal end of the Bragg peak by about 5-10\% [9]. These differences have been shown to have biologic effects, including a greater impact on paclitaxel-resistant cell lines and may translate into a greater effect on recurrent or persistent NSCLC [10]. Multiple researches have reported larger foci of DNA repair as measured by immunohistochemical staining of $\mathrm{H} 2 \mathrm{AX}$ foci following proton therapy when compared to photons $[11,12]$.

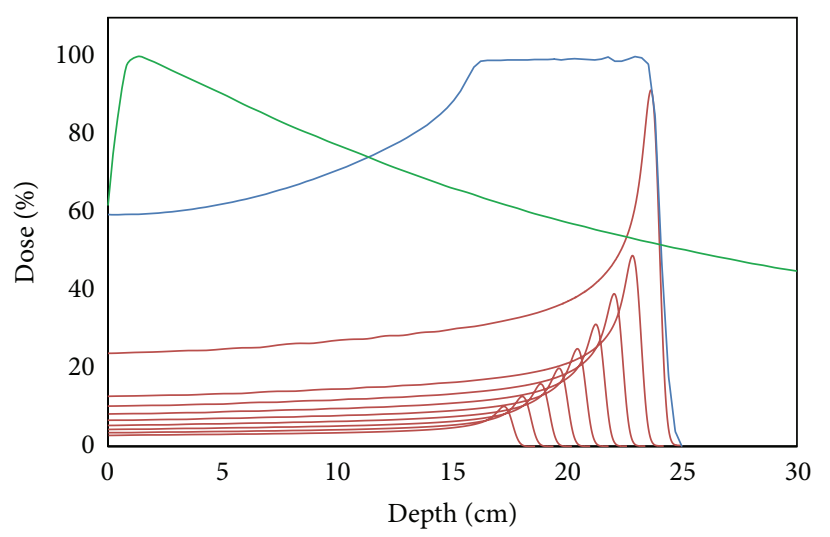

FIGURE 1: Demonstration of the Bragg peak that is characteristic of proton therapy. Individual proton beam energies are represented by the multiple red curves, with higher energies depositing their maximum energy at an increased depth. The summation of these individual beams is represented by the blue curve, known as the spread-out Bragg peak (SOBP). This SOBP is calculated and delivered such that the full depth of the target receives this maximum radiation dose. The sharp falloff following the Bragg peak allows tissues distal to the target to be spared. The green curve represents the dose deposition profile of X-ray therapy. In contrast to proton therapy, the maximum dose is deposited within several centimeters of tissue penetration and distal tissues receive a gradually decreasing amount of radiation exposure.

Protons have been shown to produce more DNA fragments [13], methylation of DNA [14], activation of free radicals [15], modulation of gene expression [16], and apoptotic activity [17]. The implication of these effects and clinical application to leverage the differences that proton therapy offers largely remain to be elucidated.

\section{Proton Therapy Delivery}

A proton is obtained by stripping a hydrogen atom of its electron, resulting in a positively charged hydrogen ion. These ions are then accelerated to a typical energy of 70-250 mega electron volts $(\mathrm{MeV})$ by a synchrotron or cyclotron. These protons are then delivered to a patient who is typically lying on a treatment table in a shielded room and directed to the appropriate depth in the body through either a "passively scattered" or "beam scanning" technique. Passively scattered proton therapy (PSPT) consists of a shaped proton beam delivered instantaneously to the patient. The lateral boundaries of the field shape are defined by passing the beam through an aperture cut out from heavy metal. A customproduced compensator is inserted into the beam path to modulate the proton depth at different points across the field. Finally, a rotating range shifter distributes the Bragg peak over the depth of the target, creating what is known as the spreadout Bragg peak (SOBP). PSPT is typically delivered with 1-4 beams in order to reduce planning uncertainties and restrict the broad deposition of radiation in the lungs [18].

In contrast, scanning beam (or intensity-modulated proton therapy, IMPT) consists of sequential targeting of 300600 spots in a voxel-like array. These spots can measure 
3-25 $\mathrm{mm}$ in size and are also known as pencil beams. The computational resources needed for this technique are significant, as each pencil beam is simultaneously optimized with inversely planned objectives. This approach allows complex targets to be treated with greater conformality, because the depth and shape of the Bragg peak are altered along the path of each beam axis. This potential for greater conformality is a double-edged sword however, with tighter margins conferring the potential for missing the target if a planning calculation differs from the actual trajectory of the beam or if the tumor moves. Technological advances to address the challenges inherent in this emerging technology and simplify the application of this emerging modality are under development $[19,20]$. A technology fundamental to photon therapy that is present in newer proton centers is on-board CT scans for verification of patient positioning at the time of treatment. The first generation of proton centers was equipped with 2-dimensional verification Xrays only for treatment verification, a limitation that will be met with newer centers and equipment upgrades. Further advances may be realized with improved accuracy in the images used to plan proton therapy. Currently, the relative proton stopping is determined from calculations based on the Hounsfield unit measurements of the planning CT scan. These calibrations introduce systematic error and are a source of range uncertainty, especially in the setting of image artifact [21]. The use of proton-based computed tomography is being developed to create an accurate electron density map for calculation of proton stopping power in the planning process [22].

\section{Motion Management and Planning Techniques}

Limiting motion and careful accounting for it in treatment planning is a hallmark of SABR. Consistency between the planning position and delivery position of the patient is especially crucial for proton-based SABR, as the specific density of tissue that each charged particle traverses determines its depth dose. If this path length is significantly altered with motion or setup variability, target miss or overtreatment of normal tissues could occur. This becomes especially important in the thorax, where tissue heterogeneity also increased uncertainties. Techniques to limit tumor motion and account for it in planning process are similar to those used in photon therapy. These include planning CT scans that characterize the path of the tumor over the entire tumor cycle (4DCT), external body frames and internal reference fiducials, compression devices, and respiratory gating systems to limit motion or synchronized radiation delivery with the breathing cycle.

Motion management is particularly important for IMPT, because the target is radiated sequentially. Intrafraction motion could thus potentially result in hot and cold spots within the tumor. To mitigate this interference between the scanning patterns and intrafractional movement, the tumor may be covered with multiple beam scans. In addition, the use of larger pencil-beam spots is more resistant to tumor motion degradation $[6,19,23]$. Adjustment for planning uncertainties in the case of PSPT is achieved using a smearing technique, in which the compensator is modified to maintain tumor coverage in the presence of small range uncertainties, at the sacrifice of some conformality.

Target volume expansions must be considered for each separate beam direction and the unique anatomic path that these protons will traverse [24]. Due to the uncertainty of tumor motion, proton range, and the lack of volumetric image guidance in most current proton centers, generous margin is typically added to ensure target coverage. This margin can be significantly reduced when fiducial tracking implants and respiratory gating are used, as on-board volumetric image guidance is introduced, a better understanding of proton range uncertainty is gained, and treatment planning is optimized, particularly for IMPT. In light of the physical properties and planning process of proton therapy, the potential clinical benefit derived from proton SABR is contingent upon a variety of patient-specific factors. These include the geometric location of critical normal structures in relation to the target, the beam angles utilized, the uncertainties associated with each beam path, and the likelihood of these reductions in normal tissue to have meaningful clinical impact in the context of the overall clinical scenario (Figure 2).

\section{Dosimetric and Clinical Studies}

A number of dosimetric studies investigating the differences in radiation dose delivered to normal tissues for proton versus photon-based SABR have been reported [6, 25-29]. These studies show the potential reduction in radiation dose to the lung, esophagus, brachial plexus, chest wall, and heart with the use of protons when compared with photon-based SABR, as shown in Table 1.

A phase II randomized clinical trial comparing proton versus photon-based SABR for centrally located or recurrent lung parenchymal early-stage NSCLC is currently ongoing in our institution. Several single-institution retrospective studies have been published and are summarized in Table 2 . Given the variability in fractionation regimens and varying definitions of what constitutes SABR, we have focused on studies that included fraction sizes greater than $4 \mathrm{~Gy}$.

Loma Linda University has the longest-term experience with proton SABR, as reported by Bush et al., where treatment was escalated from $51 \mathrm{GyE}$ to $70 \mathrm{GyE}$ in 10 fractions [30]. Local control and survival were found to improve with escalated doses. Overall survival at 5 years was $18 \%$, $32 \%$, and $51 \%$ at $51 \mathrm{GyE}, 60 \mathrm{GyE}$, and $70 \mathrm{GyE}$, respectively; however this is also subject to potential biases in overall therapeutic improvements in later time periods. Tumors greater than $5 \mathrm{~cm}$ were associated with worse local control. There were no cases of radiation pneumonitis, suggesting room for increased dose delivery to improve outcomes. PTV coverage was $3-5 \mathrm{~mm}$. As is the case here and all of the following studies, immobilization was achieved with a cradle and respiratory management was achieved with respiratory gating, $\mathrm{kV}$ imaging, and $4 \mathrm{DCT}$ in selected patients treated after 2005. 


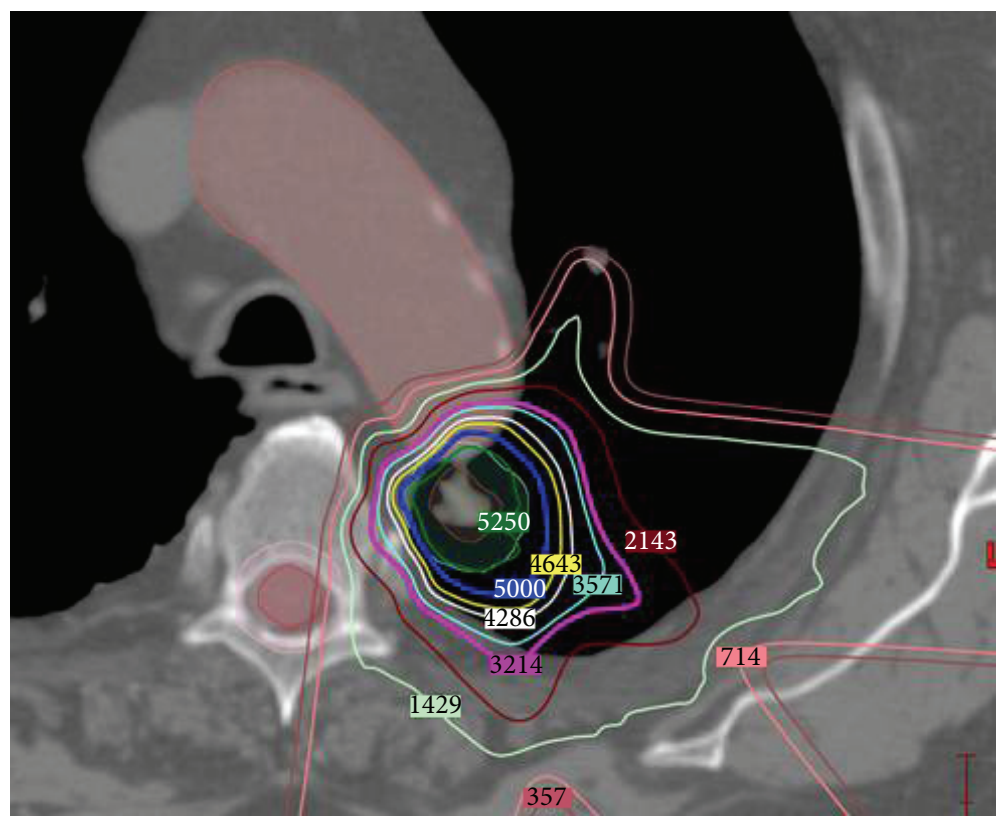

FiguRE 2: Radiation treatment plan illustrating the dosimetric benefits of proton therapy in a patient with tumor near critical central structures. Prescribed tumor dose is $50 \mathrm{GyE}$ in 4 fractions, with isodose line numbers displayed in units of cGyE. Significant radiation sparing of the aorta, esophagus, and lung is achieved due to the steep dose falloff of protons, while achieving appropriate target coverage for tumor cell kill. Beam angles are selected to traverse a minimal amount of lung tissue. Range differences based on the density heterogeneity of tissue traversed can be appreciated at the anterior aspect of the plan, where a peak of dose is deposited in normal lung. Careful attention must be paid to these dose variations and areas of range uncertainty such that dose tolerance of critical structures is not exceeded.

TABLE 1: Dosimetric reduction in normal tissue radiation for proton- versus photon-based SABR plans.

\begin{tabular}{|c|c|c|c|c|}
\hline Author, reference & $\begin{array}{l}\text { Number of plans } \\
\text { compared }(n)\end{array}$ & $\begin{array}{c}\text { Total dose, GyE } \\
\text { (dose per fraction) }\end{array}$ & $\begin{array}{l}\text { Lung reduction } \\
\text { from protons }\end{array}$ & $\begin{array}{c}\text { Esophagus } \\
\text { reduction from } \\
\text { protons } \\
\end{array}$ \\
\hline Hoppe et al., [25] & 16 & $48(12)$ & $\begin{array}{c}\text { Mean dose } 2.2 \mathrm{GyE} \\
V 5,10.4 \%^{*} \\
V 10,6.4 \% \\
V 20,2.1 \%\end{array}$ & \\
\hline Seco et al., [26] & 20 & $42(14)$ & $V 5,37.8 \%$ & $\begin{array}{c}\text { Maximum dose, } \\
68 \%\end{array}$ \\
\hline Georg et al., [27] & 36 & $45(15)$ & $\begin{array}{l}V 20, \text { PSPT, } 7-9 \% \\
V 20, \text { IMPT, }>10 \%\end{array}$ & \\
\hline Register et al., [6] & 45 & $50(12.5)$ & Mean dose, $50 \%$ & \\
\hline Kadoya et al., [29] & 21 & $66(6.6)$ & $\begin{array}{c}\text { Mean dose } 2.8 \mathrm{GyE} \\
V 5,18.8 \% \\
V 10,10.4 \% \\
V 20,2.3 \% \\
\end{array}$ & \\
\hline
\end{tabular}

${ }^{*} V x$ : percentage of structure volume receiving $\geq X$ GyE. PSPT: passively scattered proton therapy. IMPT: intensity-modulated proton therapy.

Another experience from Hata et al. included 21 patients treated with doses escalated from $50 \mathrm{GyE}$ to $60 \mathrm{GyE}$ in 10 fractions, as in the previously described study [31]. A $5 \mathrm{~mm}$ margin in the plane perpendicular to the beam axis and a $5 \mathrm{~mm}$ caudal margin were added for respiration movement. Only one local recurrence was reported at 2 years (95\% local control), with an overall survival of $74 \%$.

Nakayama et al. treated 55 patients with 58 tumors to either $66 \mathrm{GyE}$ in 10 fractions or a more protracted course for central tumors of $72.6 \mathrm{GyE}$ in 22 fractions for central tumors
[32]. This fractionated course allows for more sublethal repair of critical centrally located normal tissues. Two patients developed grade 3 pneumonitis. Planning margins included a $5 \mathrm{~mm}$ PTV for setup uncertainty, and an additional $5 \mathrm{~mm}$ margin in the caudal direction.

Nihei et al. reported 37 patients with $\mathrm{T} 1$ and $\mathrm{T} 2$ tumors below $5 \mathrm{~cm}$, treated with a total dose of 70-94 GyE in 20 fractions [33]. More than half of the patients had T2 tumors, though tumor size was limited to $<5 \mathrm{~cm}$. Two-year local control and survival were $98 \%$ and $84 \%$, respectively. Although 
TABLE 2: Summary of clinical data for proton-based stereotactic ablative and hypofractionated radiotherapy.

\begin{tabular}{|c|c|c|c|c|c|c|}
\hline Author, reference & Years & $\begin{array}{c}\text { Number of } \\
\text { cases } \\
(\mathrm{T} 1 / \mathrm{T} 2)\end{array}$ & $\begin{array}{c}\text { Total dose, GyE } \\
\text { (dose per fraction) }\end{array}$ & $\begin{array}{l}\text { Local } \\
\text { control }\end{array}$ & $\begin{array}{c}\text { Overall } \\
\text { survival }\end{array}$ & Toxicity grade $\geq 3$ \\
\hline Bush et al., [30] & Unknown & $111(47 / 64)$ & $51 / 60 / 70(5.1 / 6 / 7)$ & $\begin{array}{c}4 \mathrm{yr}, \\
45 / 75 / 86 / 91 \%^{*}\end{array}$ & $\begin{array}{c}4 \mathrm{yr} \\
18 / 32 / 51 \%^{\dagger}\end{array}$ & None \\
\hline Hata et al., [31] & 2002-2005 & $21(11 / 10)$ & $50-60(5-6)$ & $2 \mathrm{yr}, 95 \%$ & 2 yr, $74 \%$ & RP $4 \%$ \\
\hline Nakayama et al., [32] & $2001-2008$ & $58(30 / 28)$ & 66-peripheral/72.6-central $(6.6 / 3.3)$ & $2 \mathrm{yr}, 97 \%$ & $2 \mathrm{yr}, 98 \%$ & RP $4 \%$ \\
\hline Nihei et al., [33] & 1999-2003 & $37(17 / 20)$ & $70-94(3.5-4.9)$ & $2 \mathrm{yr}, 98 \%$ & 2 yr $84 \%$ & RP 8\% \\
\hline Iwata et al., [35] & 2003-2007 & $57(27 / 30)$ & $60 / 80(6 / 4)$ & $3 \mathrm{yr}, 83 / 81 \%^{\ddagger}$ & $\begin{array}{c}3 \mathrm{yr} \\
60 / 90 \%{ }^{*}\end{array}$ & RP 2\%, dermatitis 5\% \\
\hline Iwata et al., [36] & 2003-2009 & $43(0 / 43)$ & $60 / 66 / 70.2 / 80(6 / 6.6 / 2.7 / 4)$ & $3 \mathrm{yr}, 75 \%$ & $3 \mathrm{yr}, 78 \%$ & RP 3\%, dermatitis 7\% \\
\hline Fujii et al., [37] & 2003-2009 & $70(36 / 34)$ & $60 / 80(6 / 4)$ & $3 \mathrm{yr}, 81 \%$ & $3 \mathrm{yr}, 72 \%$ & $\begin{array}{l}\text { RP } 0 \% \text {, dermatitis } 4 \% \\
\text { rib fracture } 1 \% \|\end{array}$ \\
\hline Westover et al., [38] & $2008-2010$ & $20(18 / 2)$ & $42-50(10-16)$ & $2 \mathrm{yr}, 100 \%$ & $2 \mathrm{yr}, 64 \%$ & RP 7\% \\
\hline
\end{tabular}

${ }^{*}$ Reported for the following groups: (T2, $\left.60 \mathrm{GyE}\right) /(\mathrm{T} 2,70 \mathrm{GyE}) /(\mathrm{T} 1,60 \mathrm{GyE}) /(\mathrm{T} 1,70 \mathrm{GyE}),{ }^{\dagger}$ Reported for: $51 \mathrm{GyE} / 60 \mathrm{GyE} / 70 \mathrm{GyE} .{ }^{\ddagger} \mathrm{Reported}$ for $60 / 80 \mathrm{GyE}$.

${ }^{\S}$ Toxicity data includes 27 combined patients treated with carbon ion therapy, which were not separated in the manuscript. "Termed late toxicity, time period not defined. RP: radiation pneumonitis.

acute toxicities were minimal, six patients experienced late pulmonary toxicity. These late toxicities may have resulted from an excess volume of normal lung irradiated due to tumor shrinkage over the 4-week course. The importance of adaptive replanning in this context was illustrated by Chang et al., who found that $45 \%$ of patients with early-stage NSCLC treated over 7 weeks with protons therapy benefited from a repeat simulation and radiation plan due to tumor shrinkage [34]. As in the prior study, PTV margin was $5 \mathrm{~mm}$, with an additional $5 \mathrm{~mm}$ margin used caudally.

Iwata et al. and Fujii et al. have published three series of patients treated with proton and carbon ion therapy for early stage NSCLC. The first report included patients with T1-T2 tumors treated with $80 \mathrm{GyE}$ in 20 fractions or $60 \mathrm{GyE}$ in 10 fractions in the case of protons, which comprised the majority of patients [35]. Three-year local control and survival were $83 \%$ and $60 \%$, respectively, for patients treated with $60 \mathrm{GyE}$, and $81 \%$ and $90 \%$, respectively, for patients treated with $80 \mathrm{GyE}$. The second report analyzed a subset of 70 patients with T2 disease only, 43 of whom were treated with proton therapy with the addition of several different fractionation schedules [36]. Four-year local control and overall survival were $75 \%$ and $78 \%$, respectively, and no significant differences were seen between the T2a and T2b groups. Minimal toxicity was observed in patients with centrally located tumors, highlighting the potential benefit of protons for tumors with clinically challenging locations and larger size. The most recent report included 70 patients and reported a 3-year local control of $81 \%$ with overall survival at 3 years being $72 \%$ [37]. Planning margins for these studies included a $5 \mathrm{~mm}$ PTV, with a $1-4 \mathrm{~mm}$ internal margin depending on the stability of the respiration.

Westover et al. published from the most recent cohort of patients, with 20 early-stage NSCLC tumors treated with 42$50 \mathrm{GyE}$ in 3 to 5 fractions using PSPT [38]. Most of these tumors were located in a favorable position in the upper lung where breathing motion has a limited impact. Two-year
LC and OS were $100 \%$ and $64 \%$, respectively. One patient developed grade 3 pneumonitis which resolved with steroids. A $5 \mathrm{~mm}$ PTV was added, additional unspecified margin for respiratory motion, as well as 3.5\% of the proximal and distal ranges plus $2 \mathrm{~mm}$ to the proximal and distal portions of the GTV.

The above studies indicate a trend towards improved local control with more recent studies, highlighting the improving nature of this emerging technology. Recognizing the limitations of meta-analysis given the heterogeneous protocols, Grutters et al. reported the 5-year pooled overall survivals for proton therapy, carbon ion therapy, photon SABR, and conventionally-fractionated radiation to be $40 \%$, $42 \%, 42 \%$, and $20 \%$, respectively [39].

\section{Conclusions}

As the availability of proton therapy expands and the experience matures, improvements will continue to be made in the implementation of this technology. Emerging techniques such as IMPT and on-board volumetric image guidance will continue to reduce uncertainties and refine conformality [40-44]. The unique properties of protons to spare tissue distal to the target position it as a valuable tool available to the radiation oncologist for patients with critical structures near tumor, larger T2 tumors, or limited pulmonary reserve. Efforts to produce randomized data are ongoing to directly compare outcomes between photon and proton SABR. With existing clinical data, proton SABR is an effective treatment option for patients with early-stage NSCLC who may derive benefit from maximal normal tissue sparing.

\section{Conflict of Interests}

The authors declare that there is no conflict of interests regarding the publication of this paper. 


\section{Acknowledgments}

The authors would like to thank the faculty and staff of the MD Anderson Proton Center for their contributions to patient care and the research included in this paper.

\section{References}

[1] W. J. Curran, C. B. Scott, C. J. Langer et al., "Long-term benefit is observed in a phase III comparison of sequential vs concurrent chemo-radiation for patients with unresected stage III NSCLC: RTOG 9410," Proceedings of the American Society of Clinical Oncology, vol. 22, supplement 1, p. 2499, 2003.

[2] L. Potters, B. Kavanagh, J. M. Galvin et al., "American society for therapeutic radiology and oncology (ASTRO) and American college of radiology (ACR) practice guideline for the performance of stereotactic body radiation therapy," International Journal of Radiation Oncology Biology Physics, vol. 76, no. 2, pp. 326-332, 2010.

[3] I. S. Grills, V. S. Mangona, R. Welsh et al., "Outcomes after stereotactic lung radiotherapy or wedge resection for stage I non-small-cell lung cancer," Journal of Clinical Oncology, vol. 28, no. 6, pp. 928-935, 2010.

[4] H. Onishi, T. Araki, H. Shirato et al., "Stereotactic hypofractionated high-dose irradiation for stage I nonsmall cell lung carcinoma: Clinical outcomes in 245 subjects in a Japanese multiinstitutional study," Cancer, vol. 101, no. 7, pp. 1623-1631, 2004.

[5] H. Onishi, H. Shirato, Y. Nagata et al., "Hypofractionated stereotactic radiotherapy (HypoFXSRT) for stage I non-small cell lung cancer: updated results of 257 patients in a Japanese multi-institutional study," Journal of Thoracic Oncology, vol. 2, no. 7, supplement 3, pp. S94-S100, 2007.

[6] S. P. Register, X. Zhang, R. Mohan, and J. Y. Chang, "Proton stereotactic body radiation therapy for clinically challenging cases of centrally and superiorly located stage I non-smallcell lung cancer," International Journal of Radiation Oncology, Biology, Physics, vol. 80, no. 4, pp. 1015-1022, 2011.

[7] J. Y. Chang, Q.-Q. Li, Q.-Y. Xu et al., "Stereotactic ablative radiation therapy for centrally located early stage or isolated parenchymal recurrences of non-small cell lung cancer: how to fly in a "no fly zone',' International Journal of Radiation Oncology, Biology, Physics, vol. 88, no. 5, pp. 1120-1128, 2014.

[8] H. Liu, X. Zhang, Y. Y. Vinogradskiy, S. G. Swisher, R. Komaki, and J. Y. Chang, "Predicting radiation pneumonitis after stereotactic ablative radiation therapy in patients previously treated with conventional thoracic radiation therapy," International Journal of Radiation Oncology, Biology, Physics, vol. 84, no. 4, pp. 1017-1023, 2012.

[9] International Commission on Radiation Units and Measurements, "Prescribing, recording, and reporting proton-beam therapy (ICRU Report 78)," Journal of the ICRU, vol. 7, no. 2, 2007.

[10] X. Zhang, S. H. Lin, B. Fang et al., “Therapy-resistant cancer stem cells have differing sensitivity to photon versus proton beam radiation," Journal of Thoracic Oncology, vol. 8, no. 12, pp. 1484-1491, 2013.

[11] N. Finnberg, C. Wambi, J. H. Ware, A. R. Kennedy, and W. S. El-Deiry, "Gamma-radiation (GR) triggers a unique gene expression profile associated with cell death compared to proton radiation (PR) in mice in vivo," Cancer Biology and Therapy, vol. 7, no. 12, pp. 2023-2033, 2008.
[12] A. Gerelchuluun, Z. Hong, L. Sun et al., "Induction of in situ DNA double-strand breaks and apoptosis by $200 \mathrm{MeV}$ protons and 10 MV X-rays in human tumour cell lines," International Journal of Radiation Biology, vol. 87, no. 1, pp. 57-70, 2011.

[13] C. Leloup, G. Garty, G. Assaf et al., "Evaluation of lesion clustering in irradiated plasmid DNA," International Journal of Radiation Biology, vol. 81, no. 1, pp. 41-54, 2005.

[14] W. Goetz, M. N. M. Morgan, and J. E. Baulch, "The effect of radiation quality on genomic DNA methylation profiles in irradiated human cell lines," Radiation Research, vol. 175, no. 5, pp. 575-587, 2011.

[15] E. Giedzinski, R. Rola, J. R. Fike, and C. L. Limoli, "Efficient production of reactive oxygen species in neural precursor cells after exposure to $250 \mathrm{MeV}$ protons," Radiation Research, vol. 164, no. 4, pp. 540-544, 2005.

[16] J. Tian, W. Zhao, S. Tian, J. M. Slater, Z. Deng, and D. S. Gridleya, "Expression of genes involved in mouse lung cell differentiation/regulation after acute exposure to photons and protons with or without low-dose preirradiation," Radiation Research, vol. 176, no. 5, pp. 553-564, 2011.

[17] C. di Pietro, S. Piro, G. Tabbì et al., "Cellular and molecular effects of protons: apoptosis induction and potential implications for cancer therapy," Apoptosis, vol. 11, no. 1, pp. 57-66, 2006.

[18] M. Engelsman, M. Schwarz, and L. Dong, "Physics controversies in proton therapy," Seminars in Radiation Oncology, vol. 23, no. 2, pp. 88-96, 2013.

[19] C. Grassberger, S. Dowdell, A. Lomax et al., "Motion interplay as a function of patient parameters and spot size in spot scanning proton therapy for lung cancer," International Journal of Radiation Oncology Biology Physics, vol. 86, no. 2, pp. 380386, 2013.

[20] J. Y. Chang, H. Li, X. R. Zhu et al., "Clinical implementation of intensity-modulated proton therapy in thoracic malignancies," International Journal of Radiation Oncology, Biology, Physics. In press.

[21] L. de Marzi, C. Lesven, R. Ferrand, J. Sage, T. Boulé, and A. Mazal, "Calibration of CT Hounsfield units for proton therapy treatment planning: use of kilovoltage and megavoltage images and comparison of parameterized methods," Physics in Medicine and Biology, vol. 58, no. 12, pp. 4255-4276, 2013.

[22] H. F. Sadrozinski, R. P. Johnson, S. MacAfee et al., "Development of a head scanner for proton CT," Nuclear Instruments and Methods in Physics Research A: Accelerators, Spectrometers, Detectors and Associated Equipment, vol. 699, pp. 205-210, 2013.

[23] Y. Li, L. Kardar, X. Li et al., "On the interplay effects with proton scanning beams in stage III lung cancer," Medical Physics, vol. 41, no. 2, Article ID 021721, 2014.

[24] L. Widesott, M. Amichetti, and M. Schwarz, "Proton therapy in lung cancer: clinical outcomes and technical issues. A systematic review," Radiotherapy and Oncology, vol. 86, no. 2, pp. 154-164, 2008.

[25] B. S. Hoppe, S. Huh, S. Flampouri et al., "Double-scattered proton-based stereotactic body radiotherapy for stage i lung cancer: A dosimetric comparison with photon-based stereotactic body radiotherapy," Radiotherapy and Oncology, vol. 97, no. 3, pp. 425-430, 2010.

[26] J. Seco, H. R. Panahandeh, K. Westover, J. Adams, and H. Willers, "Treatment of non-small cell lung cancer patients with proton beam-based stereotactic body radiotherapy: dosimetric comparison with photon plans highlights importance of range 
uncertainty," International Journal of Radiation Oncology Biology Physics, vol. 83, no. 1, pp. 354-361, 2012.

[27] D. Georg, M. Hillbrand, M. Stock, K. Dieckmann, and R. Pötter, "Can protons improve SBRT for lung lesions? Dosimetric considerations," Radiotherapy and Oncology, vol. 88, no. 3, pp. 368-375, 2008.

[28] J. Welsh, A. Amini, K. Ciura et al., "Evaluating proton stereotactic body radiotherapy to reduce chest wall dose in the treatment of lung cancer," Medical Dosimetry, vol. 38, no. 4, pp. 442-447, 2013.

[29] N. Kadoya, Y. Obata, T. Kato et al., "Dose-volume comparison of proton radiotherapy and stereotactic body radiotherapy for non-small-cell lung cancer," International Journal of Radiation Oncology Biology Physics, vol. 79, no. 4, pp. 1225-1231, 2011.

[30] D. A. Bush, G. Cheek, S. Zaheer et al., "High-dose hypofractionated proton beam radiation therapy is safe and effective for central and peripheral early-stage non-small cell lung cancer: Results of a 12-year experience at loma linda university medical center," International Journal of Radiation Oncology Biology Physics, vol. 86, no. 5, pp. 964-968, 2013.

[31] M. Hata, K. Tokuuye, K. Kagei et al., "Hypofractionated highdose proton beam therapy for stage I non-small-cell lung cancer: preliminary results of a phase I/II clinical study," International Journal of Radiation Oncology Biology Physics, vol. 68, no. 3, pp. 786-793, 2007.

[32] H. Nakayama, S. Sugahara, M. Tokita et al., "Proton beam therapy for patients with medically inoperable stage I non-small-cell lung cancer at the University of Tsukuba," International Journal of Radiation Oncology Biology Physics, vol. 78, no. 2, pp. 467-471, 2010.

[33] K. Nihei, T. Ogino, S. Ishikura, and H. Nishimura, "High-dose proton beam therapy for Stage I non-small-cell lung cancer," International Journal of Radiation Oncology Biology Physics, vol. 65, no. 1, pp. 107-111, 2006.

[34] J. Y. Chang, R. Komaki, H. Y. Wen et al., "Toxicity and patterns of failure of adaptive/ablative proton therapy for early-stage, medically inoperable non-small cell lung cancer," International Journal of Radiation Oncology Biology Physics, vol. 80, no. 5, pp. 1350-1357, 2011.

[35] H. Iwata, M. Murakami, Y. Demizu et al., "High-dose proton therapy and carbon-ion therapy for stage I nonsmall cell lung cancer," Cancer, vol. 116, no. 10, pp. 2476-2485, 2010.

[36] H. Iwata, Y. Demizu, O. Fujii et al., "Long-term outcome of proton therapy and carbon-ion therapy for large (T2aT2bN0M0) non-small-cell lung cancer," Journal of Thoracic Oncology, vol. 8, no. 6, pp. 726-735, 2013.

[37] O. Fujii, Y. Demizu, N. Hashimoto et al., "A retrospective comparison of proton therapy and carbon ion therapy for stage I non-small cell lung cancer," Radiotherapy and Oncology, vol. 109, no. 1, pp. 32-37, 2013.

[38] K. D. Westover, J. Seco, J. A. Adams et al., "Proton SBRT for medically inoperable stage i NSCLC," Journal of Thoracic Oncology, vol. 7, no. 6, pp. 1021-1025, 2012.

[39] J. P. C. Grutters, A. G. H. Kessels, M. Pijls-Johannesma, D. De Ruysscher, M. A. Joore, and P. Lambin, "Comparison of the effectiveness of radiotherapy with photons, protons and carbon-ions for non-small cell lung cancer: a meta-analysis," Radiotherapy and Oncology, vol. 95, no. 1, pp. 32-40, 2010.

[40] J. Seco, G. Gu, T. Marcelos, H. Kooy, and H. Willers, "Proton arc reduces range uncertainty effects and improves conformality compared with photon volumetric modulated arc therapy in stereotactic body radiation therapy for non-small cell lung cancer," International Journal of Radiation Oncology Biology Physics, vol. 87, no. 1, pp. 188-194, 2013.

[41] D. De Ruysscher and J. Y. Chang, "Clinical controversies: proton therapy for thoracic tumors," Seminars in Radiation Oncology, vol. 23, no. 2, pp. 115-119, 2013.

[42] A. J. Lomax, "Intensity modulated proton therapy and its sensitivity to treatment uncertainties 1 : the potential effects of calculational uncertainties," Physics in Medicine and Biology, vol. 53, no. 4, pp. 1027-1042, 2008.

[43] W. Liu, X. Zhang, Y. Li, and R. Mohan, "Robust optimization of intensity modulated proton therapy," Medical Physics, vol. 39, no. 2, pp. 1079-1091, 2012.

[44] M. Stuschke, A. Kaiser, C. Pöttgen, W. Lübcke, and J. Farr, "Potentials of robust intensity modulated scanning proton plans for locally advanced lung cancer in comparison to intensity modulated photon plans," Radiotherapy and Oncology, vol. 104, no. 1, pp. 45-51, 2012. 


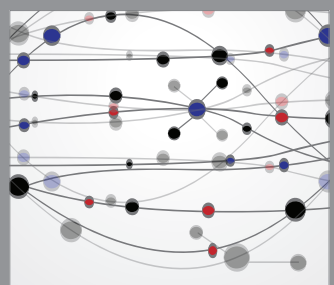

The Scientific World Journal
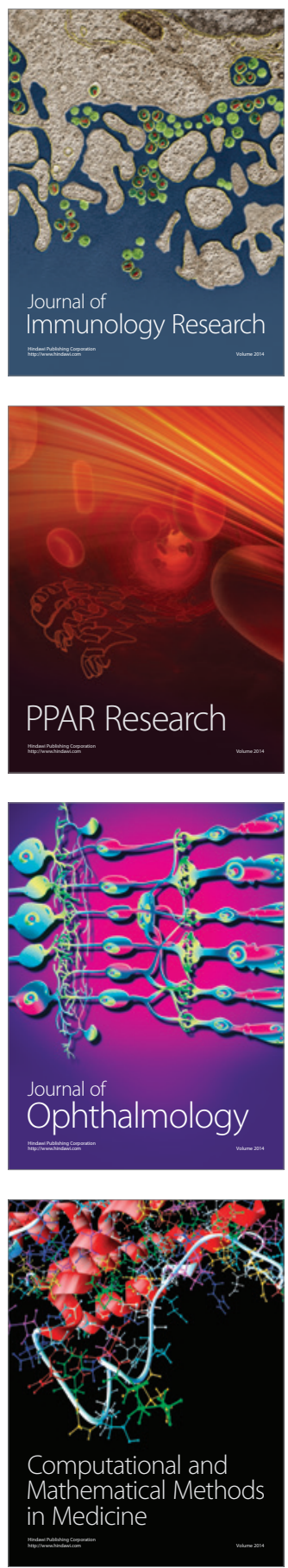

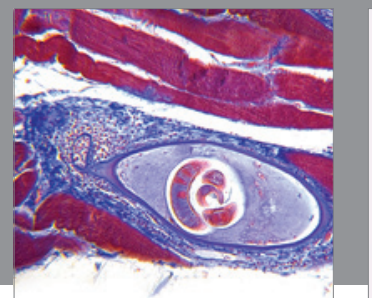

Gastroenterology

Research and Practice
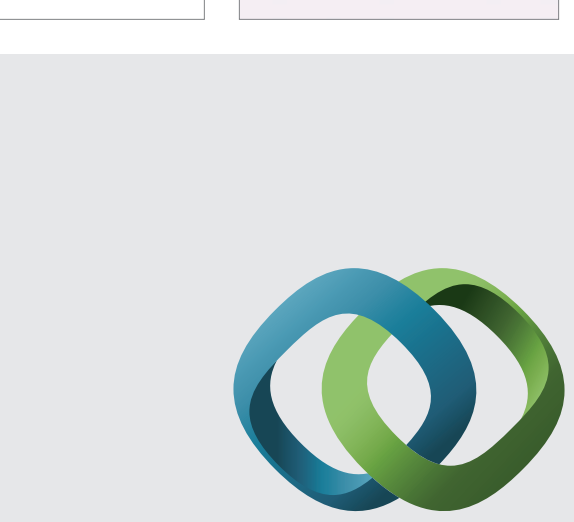

\section{Hindawi}

Submit your manuscripts at

http://www.hindawi.com
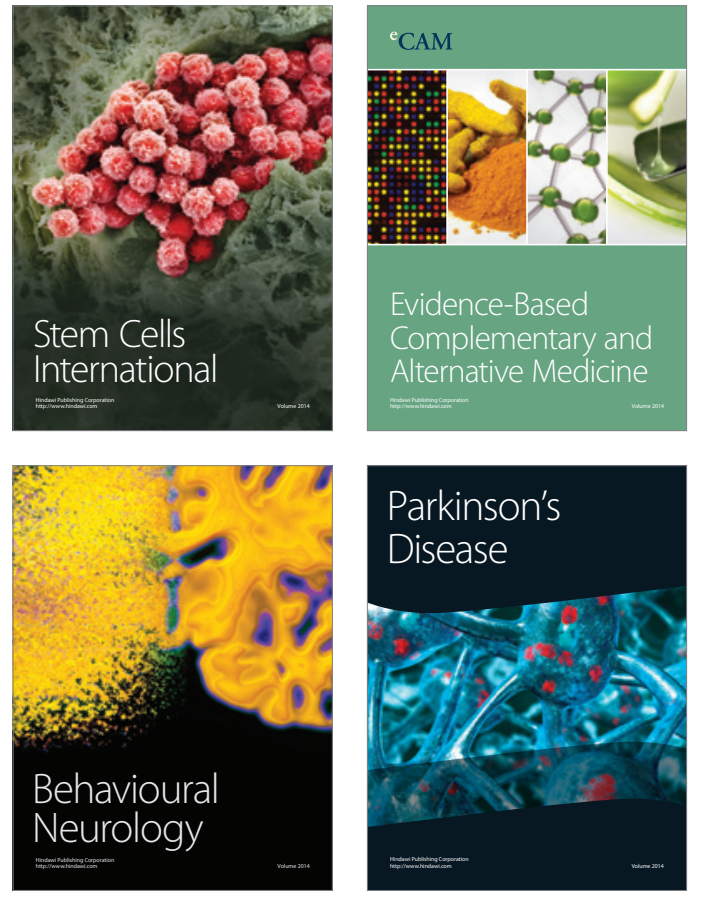
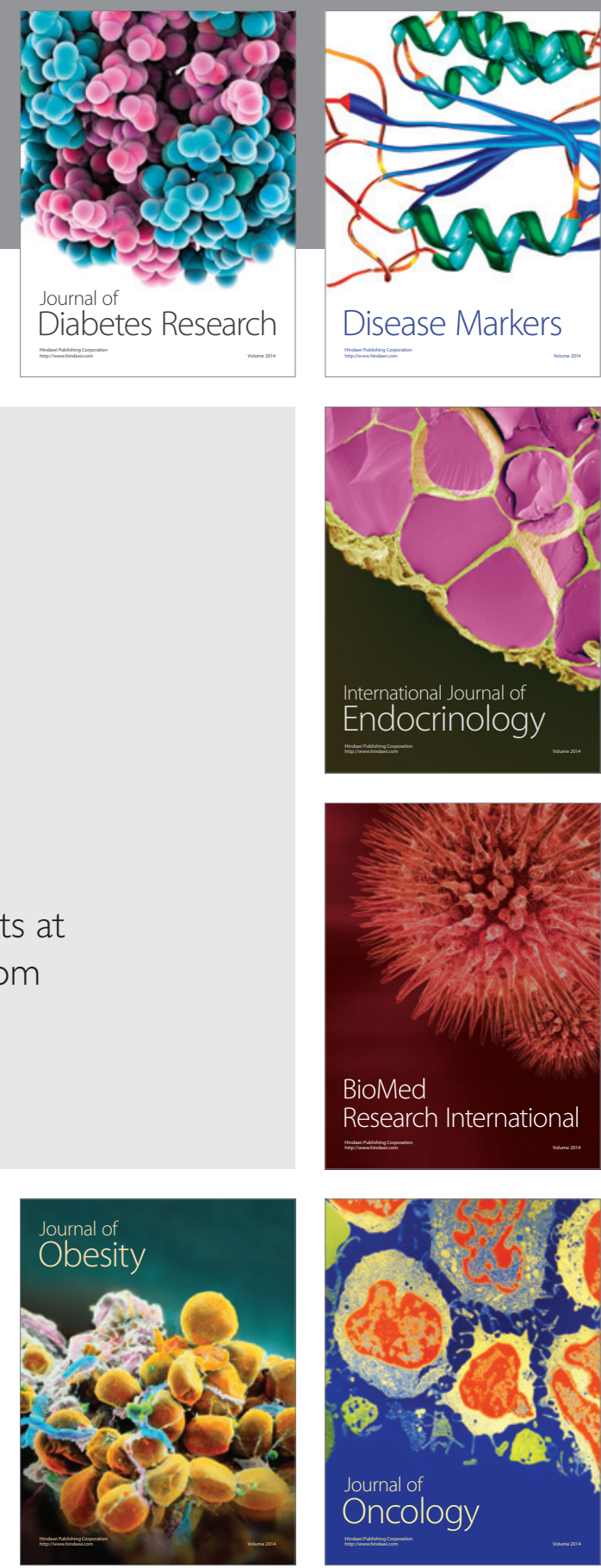

Disease Markers
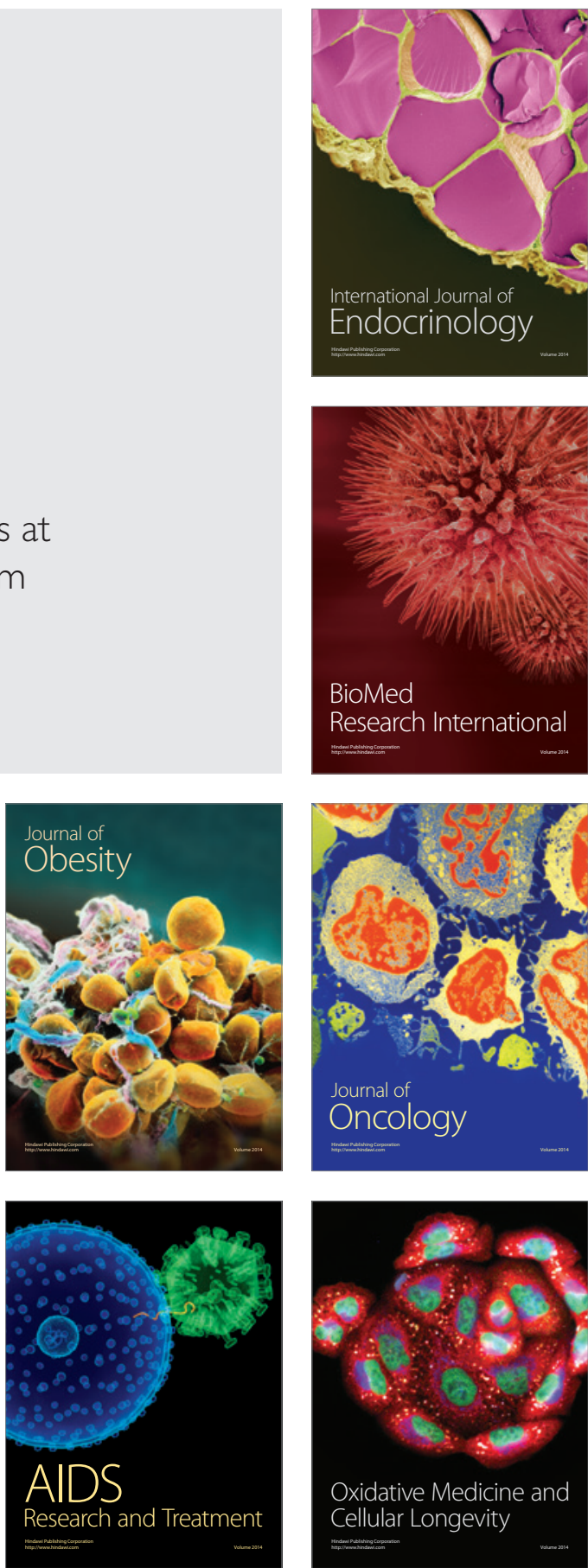